Methods Selected community health workers (CHWs) were initially trained for simple malaria management among children under five years old using artemisinin-based combination therapy (ACT) and to perform rapid diagnostic tests for malaria (RDTs), and then equipped to provide service. This was a retrospective and prospective study using a mixed-methods approach between September-October 2017. Data before CCMM initiation (2013-2014) were collected from the Kabonga health centre, then compared to data from 20152016 corresponding to CCMM implementation. Data were from health records and stakeholders and were processed using SPSS.

Results In total, 5922 children were treated for malaria between 2013-2014 and 5249 between 2015-2016, an 11,3\% case decrease. CHWs managed in total 1751 children using ACTs between 2015-2016. Of them, 1297 (74\%) had a consult within 24 hours of fever onset and the cure rate was approximately 90\%. Mortality decreased with up to $75 \%$. Ownership level was encouraging among beneficiary communities and was low among district and health center's staff. Some stock-outs were also reported.

Conclusion Using trained CHWs increased access to timely and appropriate treatment in sampled communities which was associated with improved health outcomes among children under 5 years old. However, regular supply to CHWs and strong ownership is needed.

\section{PO 8468 DETERMINE TB-LAM LATERAL FLOW ASSAY: DOES M. TUBERCULOSIS LINEAGE INFLUENCE ITS PERFORMANCE?}

${ }^{1}$ Willy Ssengooba*, ${ }^{2}$ Lydia Nakiyingi, ${ }^{1}$ Joloba Moses. 'Department of Medical Microbiology, College of Health Sciences Makerere University Kampala, Uganda; ${ }^{2}$ Infectious Diseases Institute, College of Health Sciences Makerere University, Kampala, Uganda

\subsection{6/bmjgh-2019-EDC.107}

Background Among HIV-positive individuals with CD4 $\leq 100$ cells $/ \mathrm{mm}^{3}$, M. tuberculosis (Mtb) bacteraemia is associated with a positive tuberculosis urine lipoarabinomannan (LAM) test. We conducted a retrospective study, to determine the effect of Mtb lineage on the performance of the Determine TB LAM lateral flow (LF-LAM) assay.

Methods This was nested in a prospective TB diagnostics accuracy study among HIV-positive presumptive TB patients from Mulago National Referral Hospital, Kampala, Uganda, including both inpatients and outpatients. We considered data of 51 HIV-positive individuals with both pulmonary and Mtb bacteraemia. We also evaluated the effect of having mixed $\mathrm{Mtb}$ strains using both spoligotyping and MIRU-VNTR 24 loci methods.

Results LF-LAM was; negative among 4 (7.8\%; 95\% CI, $2.17 \%$ to $18.8 \%)$, positive among $39(76.5 \%$; $95 \% \mathrm{CI}, 62.5 \%$ to $87.2 \%)$ and indeterminate among $8(15.7 \%$; $95 \% \mathrm{CI}, 7.0 \%$ to $28.5 \%$ ) participants. Mtb lineages from blood samples were: Central Asian Strain (CAS; L3) 10/51 (19.6\%) and Euro-American lineage (L4) 41/51 (80.4\%). Mtb lineages from sputum samples were $7 / 51$ (13.7\%) L3 and 44/51 (86.3\%) were L4. Among participants with L3 in blood, LFLAM was positive in 9 (90\%; 95\% CI, 55.4\%-99.7\%) whereas those with L4, LF-LAM was positive among 30 (73.2\% 95\% CI, $57.0 \%$ to $85.7 \%$ ). For those with L3 in sputum, LF-LAM was positive among $7(100 \%)$ and those with L4 was $32(72.7 \%$;
95\% CI $57.2 \%$ to $85.0 \%$ ). Two participants had mixed Mtb strains (all L4) and all LF-LAM positive $(2+$ and $4+$ ).

Conclusion Our study shows that $M$. tuberculosis lineage 3 may have more sensitivity to LF-LAM assay than lineage 4 . The high number of indeterminate results with L4 requires more investigations. Our findings suggest that LF-LAM performance may differ by geographical regions depending on the dominant M. tuberculosis lineage.

\section{PO 8470 FIRST INDEPENDENT ASSESSMENT OF PHARMACEUTICAL COMPANY ACTION ON AMR}

Jacqueline Kisakye, Josefien Knoeff, Adrian Ruiz, Dulce Calcada, Jayasree Iyer, J Gabrielle Breugelmans. Access to Medicine Foundation, Amsterdam, The Netherlands

\subsection{6/bmjgh-2019-EDC.108}

Background Antimicrobial resistance (AMR) is one of the most significant threats to public health globally. It will worsen without concerted efforts to spur the development of new antibiotics and ensure access and stewardship of existing ones. Pharmaceutical companies have a critical role to play in these efforts. The Access to Medicine Foundation developed the AMR Benchmark to measure how pharmaceutical companies are responding to AMR and to share and push best practices within the industry.

Methods The AMR Benchmark assessed the AMR activities of 30 pharmaceutical companies in 106 low- and middle-income countries. Survey data on company activities were collected across three research areas: research and development (R\&D); manufacturing and production; and access and stewardship. For each research area, specific metrics were developed to evaluate company performance. These metrics were defined through consultation with experts working across the AMR field and represent a broad consensus on where companies can and should be taking action to limit AMR.

Results The Benchmark found that there are good practices in all research areas. Out of 276 R\&D projects targeting infectious diseases, 175 target pathogens identified as priority by WHO/CDC. Of these, 88 are in preclinical stage, 87 are in clinical stage, and 54 target gram-negative bacteria. Out of 28 antibiotics in late clinical stage, only two have access and stewardship plans in place. Nearly half of companies evaluated are involved in AMR surveillance and eight companies set limits on antibiotic wastewater discharge. Lastly, four companies separate sales agent bonuses from antibiotics sales volumes to reduce overuse of antibiotics.

Conclusion The Benchmark identified the good ideas being implemented by companies to limit AMR and mapped opportunities to amplify current efforts. Although companies are taking some action, the R\&D pipeline needs to be further strengthened and candidates reaching late clinical stage must be supported by concrete plans to ensure access and stewardship.

\section{PO 8471 A SURVEY OF CURRENT CLINICAL DATA MANAGEMENT PRACTICES IN SUB-SAHARAN AFRICA}

${ }^{1}$ Hanne Landuyt* , ${ }^{1}$ Harry Van Loen, ${ }^{2}$ Bai L Dondeh, ${ }^{2}$ Badou M Gaye, ${ }^{1}$ Yven Van Herrewege. ${ }^{1}$ Institute of Tropical Medicine, Antwerp, Belgium; ${ }^{2}$ Medical Research Council Unit The Gambia at the London School of Hygiene and Tropical Medicine, London, UK

10.1136/bmjgh-2019-EDC.109 